\title{
Os Mestrados Profissionais em Ensino de Ciências e Matemática no Brasil: um tema pouco explorado na literatura.
}

Paulo Vinícius Rebeque paulo.rebeque@bento.ifrs.edu.br 0000-0002-2830-9435 Grande do Sul, ento Gonçalves, Brasil

Fernanda Ostermann

$\frac{\text { fernanda@if.ufrgs.br }}{0000-0002-0594-2174}$

Universidade Federal do Rio Grande do Sul, Porto Alegre, Brasil.

\section{Sofia Viseu}

sviseu@ie.ulisboa.pt

Universidade de Lisboa, Lisboa, Portugal

\author{
RESUMO
}

Neste artigo apresentamos uma revisão da produção acadêmica sobre os cursos de Mestrados Profissionais em Ensino de Ciências e Matemática (MPECM) no Brasil. Para tal, examinamos, em acervos digitais, todo material publicado na última década em 20 periódicos nacionais e internacionais e no Encontro Nacional de Pesquisa em Educação em Ciências (ENPEC). Dentro do grande volume de textos consultados, nossos achados sobre o MPECM foram de 37 publicações, indicando que o tema ainda é pouco explorado na literatura. A presente revisão foi importante para identificarmos tendências em estudos que caracterizam os trabalhos de conclusão produzidos nos MPECM e em investigações sobre cursos específicos de Física e Matemática, mas não sobre cursos de Biologia e Química. Por outro lado, notadamente, há uma carência de pesquisas sobre as diretrizes e os currículos dos MPECM, sobre os recentes programas em Rede Nacional e sobre o papel do MPECM como instrumento de política pública de formação continuada de professores.
\end{abstract}

PALAVRAS-CHAVE: Mestrado Profissional. Ensino de Ciências e Matemática. Formação continuada de professores. 


\section{INTRODUÇÃO}

Em Outubro de 1995 a Coordenação de Aperfeiçoamento de Pessoal de Nível Superior (CAPES), por meio da Portaria $n^{\circ} .47 / 1995$ (CAPES, 1995), determinou a implantação de procedimentos apropriados à recomendação, acompanhamento e avaliação de cursos de mestrados dirigidos à formação profissional ${ }^{1}$. Passou a existir, então, duas modalidades de mestrado na pós-graduação brasileira: Mestrado Profissional (MP) e Mestrado Acadêmico (MA).

No âmbito da formação continuada de professores, surgiu em 2002 os primeiros cursos de Mestrado Profissional em Ensino (MPE). Com objetivo diferente do MA, concentrado na iniciação de um pesquisador (etapa que precede o doutorado), o MPE é direcionado para uma qualificação da formação didáticopedagógica de professores que atuam na educação básica em uma específica área do conhecimento: trata-se de uma formação profissional que prepara o docente para ministrar disciplinas de conteúdos específicos (MOREIRA, 2004).

Nesse cenário de distinção entre MPE e MA dedicados ao ensino, a então área de Ensino de Ciência e Matemática (ECM) - criada em Setembro de 2000 no sistema de avaliação da CAPES e inserida na área de Ensino como subárea a partir da Portaria $\mathrm{n}^{\circ}$. 83, de 6 de Junho de 2011 (BRASIL, 2011) -, apresentou em Outubro de 2002 documentos sobre as características dos MA e dos MPE e os critérios de avaliação para cada modalidade (MOREIRA, 2002 e CAPES, 2007). A partir de então, esta modalidade de mestrado ganhou grande projeção no Sistema Nacional de Pós-Graduação (SNPG)2 , sobretudo com a criação dos cursos de Mestrado Profissional em Ensino de Ciências e Matemática (MPECM).

Ainda nesta época, Moreira (p. 42, 2002) apresentou uma preocupação quanto ao crescimento da área de ECM na pós-graduação, afirmando que a mesma não poderia crescer apenas na direção dos cursos de MPECM: "eles são importantes como alternativa de pós-graduação stricto sensu para professores, mas o fortalecimento da Área depende muito dos mestrados acadêmicos e sobretudo de mais programas de doutorado".

Porém, o que constatamos nos primeiros anos de existência da área de ECM foi uma expressiva expansão de cursos de mestrados na modalidade profissional, de modo que em apenas setes anos estes cursos já superavam em quantitativo os cursos de MA na área de ECM (CAPES, 2010), conforme ilustra a Figura 1 (a). A partir da criação da área de Ensino (BRASIL, 2011), os cursos da antiga área de ECM passaram a ser contabilizados com cursos de outras áreas do conhecimento, mas que também se dedicam ao ensino, tais como: Ensino de Ciências da Saúde (Saúde Coletiva, Educação e Saúde na Infância e Adolescência) e Ensino (Humanidades, Linguagens, Educação Básica). Assim, conforme Figura 1 (b), o quantitativo de cursos de mestrados direcionados ao ensino continuou a crescer com maior intensidade na modalidade profissional (CAPES, 2013).

Figura 1 - Evolução quantitativa dos cursos de mestrado (a) na área de Ensino de Ciências e Matemática (Set/2000 - Jun/2011) e (b) na área de Ensino (Jan/2012 - Jan/2017). 


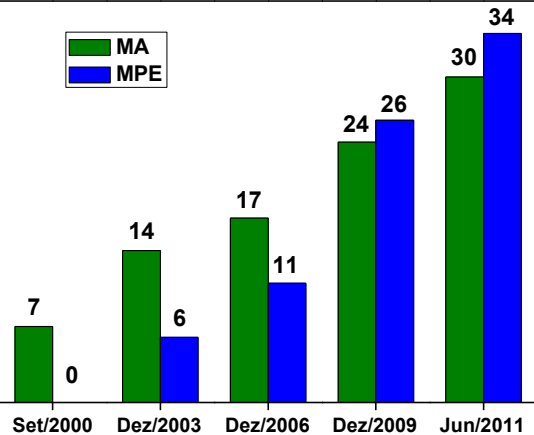

(a)

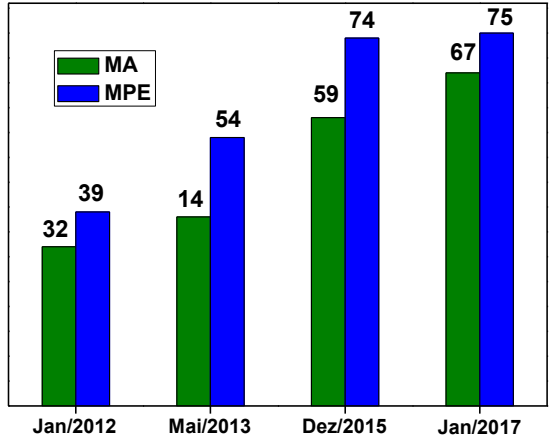

(b)

As figuras acima revelam que a implantação de cursos de MPE tem sido uma forte política da CAPES, justificada, segundo essa agência, pela necessidade de enfrentar os "debates sobre estratégias e mecanismos de qualificação de professores em larga escala" (CAPES, p. 9-10, 2013). Tal fato é perceptível quando olhamos para as dez áreas de avaliação do SNPG que possuem maior quantitativo de cursos de mestrados: a área de Ensino é a sétima maior em oferta de cursos de mestrados, mas a terceira com maior quantitativo de cursos na modalidade profissional e a única em que estes cursos superam os cursos acadêmicos.

Tabela 1 - quantitativo de cursos de mestrado nas dez áreas de avaliação do SNPG com maior oferta de cursos neste nível de pós-graduação (Mar/2017).

\begin{tabular}{|c|c|c|c|c|c|c|c|c|c|c|}
\hline & 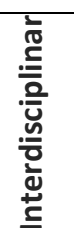 & 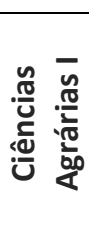 & 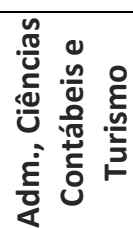 & 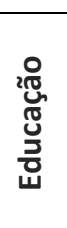 & 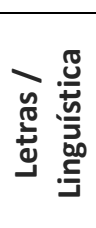 & $\begin{array}{l}\frac{0}{0} \\
\frac{\pi}{0} \\
\frac{0}{0} \\
\frac{0}{0} \\
\frac{2}{0} \\
\frac{0}{0} \\
\frac{0}{0}\end{array}$ & 号 & 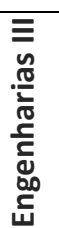 & 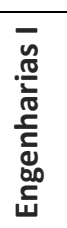 & 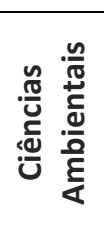 \\
\hline MA & 240 & 204 & 109 & 128 & 146 & 139 & 67 & 98 & 98 & 84 \\
\hline MP & 98 & 20 & 75 & 44 & 9 & 6 & 75 & 30 & 24 & 33 \\
\hline Total & 338 & 224 & 184 & 172 & 155 & 145 & 142 & 128 & 122 & 117 \\
\hline
\end{tabular}

Como mencionamos, os 142 cursos de mestrados que compõem a área de Ensino podem ser divididos, grosso modo, em três subáreas: Ensino de Ciências e Matemática, Ensino de Ciências da Saúde e Ensino. Essa subdivisão nos evidencia a superioridade dos cursos de mestrado em ECM com aproximadamente $67 \%$ do total (dos quais 43 são cursos de MA e 50 são cursos de MPE), sendo os $33 \%$ restantes divididos, praticamente em proporção igual, entre os cursos de mestrado em Ensino de Ciências da Saúde e em Ensino.

De fato, a oferta dos cursos de MPECM é maior que a dos os cursos acadêmicos desde 2009, sete anos após a criação dos primeiros cursos de MPE. Vale lembrar que, diferente da antiga área de ECM que no decorrer de sua trajetória passou a dar prioridade à criação de cursos de MPE em relação aos cursos de MA, a área de Ensino já no momento de sua criação registrava um quantitativo maior de cursos de MPE. Por isso não há exagero em dizermos que a superioridade dos cursos de MPE na área de Ensino nada mais é do que uma "herança" da antiga área de ECM. 
Tal quadro tem sido alvo de debates, discussões, posições antagônicas e disputas políticas de influência e controle entre grupos no meio acadêmico (VILLANI, 2016). Mesmo porque, a superioridade dos MPE indica o caminho que a pós-graduação em Ensino tem priorizado: o da formação didático-pedagógica de professores que atuam na educação básica.

Nesse contexto, passados quinze anos de criação dos primeiros cursos de MPECM, parece-nos um momento bastante oportuno para mapearmos a produção acadêmica na área de Ensino e trazer à tona elementos que possam contribuir para uma reflexão sobre o tema. O estudo que aqui apresentamos, de caráter exploratório, consistiu na procura e seleção de pesquisas acadêmicas sobre - MPECM divulgadas em periódicos e atas de eventos para, então, analisá-las de modo a destacar os principais temas abordados.

\section{MÉTODO DE BUSCA E ANÁLISE}

Nossa revisão da produção acadêmica divulgada em periódicos e anais de eventos partiu da problemática dos cursos de MPECM, sendo realizada sobre as publicações da última década (2007 - 2016) em 20 revistas nacionais e internacionais - extratos A1, A2 ou B1 da área de Ensino, segundo classificação da CAPES 2014 - e nas Atas do Encontro Nacional de Pesquisa em Educação em Ciências (ENPEC), principal evento de pesquisa acadêmica em ECM do Brasil.

Os textos sobre o MPECM foram selecionados mediante leitura do título, do resumo e das palavras-chave de todo material consultado em acervo digital ${ }^{3}$. Nos textos em português buscamos os descritores "Mestrado Profissional em Ensino de Ciências (e Matemática)" e "formação (continuada) de professores", enquanto que nos textos em inglês e espanhol procuramos por "professional master's degrees in Science (and Math) teaching", "(in service) teacher education" e "maestría en la Enseñanza de las Ciencias (y las Matemáticas)", "formación (contínua) del professorado", respectivamente.

Importa-nos esclarecer que há várias publicações que relatam o processo de construção e aplicação de produtos educacionais oriundos de cursos de MPECM. Trabalhos dessa natureza não estão presentes em nossos achados, pois, além de já estarem publicados como trabalho de conclusão (dissertação e produto educacional), não se concentram, necessariamente, na pesquisa sobre os MPECM. Como exemplo, temos a edição especial do Boletim de Educação Matemática (v. 27, n. 46, 2013) que publicou 21 artigos de produtos educacionais desenvolvidos em MPECM ou MP em Educação Matemática e aplicados nos mais diversos contextos educacionais. Ou ainda, os trabalhos de Wolff e Mors (2006), Krummenauer, Costa e Silveira (2010), Silva Neto, Ostermann e Prado (2011) e Oliveira, Veit e Araujo (2015): exemplos de produtos educacionais elaborados no âmbito do Mestrado Profissional em Ensino de Física (MPEF) da Universidade Federal do Rio Grande do Sul (UFRGS).

De todo material consultado, encontramos 37 publicações sobre o MPECM. Assim, realizamos uma análise interpretativa dessas publicações, de modo a identificar o tema principal explorado em cada uma delas. Isso nos permitiu separar o material analisado nos seguintes grupos: (a) aspectos gerais do MPE e do MA na área de ECM, (b) identificação e categorização de dissertações e produtos educacionais, (c) entrevistas com egressos de MPECM, (d) contribuições 
e potencialidades dos MPECM para a formação continuada de professores, (e) discussão sobre os Mestrados Profissionais em Rede Nacional e (f) investigações de vários aspectos no contexto específico de cursos de MPECM.

As tabelas 2 e 3 apresentam, respectivamente, informações sobre os periódicos e sobre as atas consultadas em nossa revisão bibliográfica.

Tabela 2 - Lista dos periódicos consultados, ISSN (International Standard Serial Number) e número de artigos encontrados sobre cursos de MPECM.

\begin{tabular}{lll}
\hline \multicolumn{1}{c}{ Nome do periódico } & ISSN & Artigos \\
\hline Alexandria: Revista de Educação em Ciência e Tecnologia & $1982-5153$ & 0 \\
Avaliação: revista da avaliação da educação superior & $1982-5765$ & 0 \\
Boletim de Educação Matemática & $1980-4415$ & 3 \\
Caderno Brasileiro de Ensino de Física & $2175-7941$ & 2 \\
Ciência \& Educação & $1980-850 X$ & 4 \\
Ensaio: pesquisa em educação em ciências & $1983-2117$ & 2 \\
Experiências em Ensino de Ciências & $1982-2413$ & 1 \\
International Journal of Science Education & $1464-5289$ & 0 \\
Investigações em Ensino de Ciências & $1518-8795$ & 0 \\
Revista Brasileira de Educação & $1809-449 X$ & 1 \\
Revista Brasileira de Ensino de Ciência e Tecnologia & $1982-873 X$ & 3 \\
Revista Brasileira de Ensino de Física & $1806-9126$ & 0 \\
Revista Brasileira de Ensino de Química & $1809-6158$ & 0 \\
Revista Brasileira de Pesquisa em Educação em Ciências & $1984-2686$ & 0 \\
Revista Brasileira de Pós-Graduação & $2358-2332$ & 3 \\
Revista de Educación en Biología & $2344-9225$ & 0 \\
Revista Electrónica de Enseñanza de las Ciencias & $1579-1513$ & 1 \\
Revista Electrónica de Investigación en Educación en Ciências & $1850-6666$ & 0 \\
Revista Lusófona de Educação & $1646-401 X$ & 0 \\
Science \& Education & $1573-1901$ & 0 \\
\hline & &
\end{tabular}

Tabela 3 - Lista das Atas do ENPEC consultadas e número de trabalhos encontrados sobre os cursos de MPECM.

\begin{tabular}{lc}
\hline \multicolumn{1}{c}{ Edição do ENPEC, ano e cidade de realização } & Trabalhos \\
\hline X ENPEC, 2015, Águas de Lindóia / SP & 9 \\
IX ENPEC, 2013, Águas de Lindóia / SP & 2 \\
VIII ENPEC, 2011, Campinas / SP & 3 \\
VII ENPEC, 2009, Florianópolis / SC & 1 \\
VI ENPEC, 2007, Florianópolis / SC & 2 \\
\hline
\end{tabular}

\section{RESULTADOS}

Nossos achados revelam que, passados quinze anos de criação dos primeiros cursos de MPECM, a produção acadêmica sobre o tema ainda é incipiente, apresentando um total de 37 publicações: 20 artigos nas revistas consultadas e 17 trabalhos completos nas últimas cinco edições do ENPEC.

A seguir, apresentamos uma síntese das publicações selecionadas de acordo com os grupos identificados. 


\section{(a) aspectos gerais do MPE e do MA na área de ECM}

Esse grupo é formado por quatro artigos que, de algum modo, apresentam e debatem características das duas modalidades de mestrado em ECM.

Em função da recorrente confusão entre MPE e MA na então área de ECM, Moreira e Nardi (2009) apresentaram, a partir de documentos gerados pelas comissões e coordenações desta área, esclarecimentos relativos sobre os MPECM, chamando a atenção de que este não é um documento oficial da área. Ao longo do texto, os autores deixam claro que o MPECM é uma proposta nova, não uma adaptação do MA, e explicam a natureza, o público-alvo, as atividades didáticas (de conteúdo e de caráter pedagógico), o trabalho de conclusão (dissertação e o produto educacional), a avaliação, entre outras características.

Já Carneiro (2008) coloca que devemos, em primeiro lugar, compreender que o que se espera de um professor em formação nos MPECM não é uma "pesquisa acadêmica ou científica", mas sim o que se denomina como "pesquisa do professor" (algo próximo da expressão "pesquisa profissional aplicada" utilizada para os cursos de MPE): intimamente relacionada à prática docente, uma vez que busca o conhecimento da realidade escolar cotidiana para promover práticas pedagógicas transformadoras. Com essa diferenciação devidamente estabelecida, a autora apresenta critérios de qualidade e desenvolve reflexões sobre caminhos para a formação de professores de Matemática inseridos nos MPECM.

Quanto à natureza da pesquisa para cada modalidade de mestrado em ECM, Ostermann e Rezende (2009) explicam que enquanto no MA o foco são pesquisas básicas sobre o Ensino, as pesquisas desenvolvidas nos MPE, por serem restritas à aplicação de atividades didáticas, devem ser construídas a partir de questões-foco, ou seja, devem estar relacionadas "a formas de se conceber, implementar e avaliar inovações didáticas (estando vinculadas a metodologias de ensino, conteúdos e avaliação)" (OSTERMANN e REZENDE, p. 72, 2009). Para as autoras, os produtos educacionais não podem ser entendidos como um instrumento que, quando aplicado na escola, "facilita" a aprendizagem dos alunos. Essa visão tecnicista do ensino deve ser superada por reflexões mais amplas e que envolvam os problemas enfrentados nos diversos contextos escolares.

O papel do MPECM e suas possíveis contribuições para a melhoria da educação de nível médio, em particular, da educação em Ciências, é o cerne das reflexões empreendidas por Rezende e Ostermann (2015). A partir de um estudo crítico da atual situação do Ensino Médio no país e da formação de professores, as autoras analisaram aspectos curriculares e estruturais dos cursos de MPECM de modo a concluírem que "o protagonismo dos MP em ensino em relação à qualidade da educação de nível médio é, no mínimo, controverso, isto é, não é algo que se possa tomar como garantido" (REZENDE e OSTERMANN, p. 556, 2015).

\section{(b) identificação e categorização de dissertações e produtos educacionais}

Nos cursos de MPE é necessário desenvolver "um processo ou produto educativo e utilizá-lo em condições reais de sala de aula ou de espaços não-formais ou informais de ensino" (CAPES, 2013, p. 24 -25), sendo que o relato desta experiência (desenvolvimento, aplicação e avaliação) deve ser descrito na tomaram como objeto de estudo essas produções. 
Ao analisarem 54 dissertações defendidas no período de 2004 a 2009 no MPE de Ciências Naturais e Matemática da Universidade Federal do Rio Grande do Norte (UFRN), Carvalho et al. (2011) verificaram que desse total oito não geraram produto educacional, pois se dedicaram à análise crítica de livros didáticos. As demais dissertações geraram produtos nas seguintes disciplinas: um em Ciências, dois em Biologia, quatro em Química, 18 em Física e 23 em Matemática. No entanto, os autores evidenciaram o pequeno poder de penetração (e continuidade de estudos) dessas produções e experiências no ambiente escolar.

De acordo com Santos et al. (2012), das dissertações defendidas entre 2009 e 2010 no MPE de Ciências da Universidade do Grande Rio (UNIRIO) que abordavam a questão didática mediada pelas tecnologias da informação e comunicação (TIC), apenas quatro se enquadraram nessas especificações. Ao examiná-las, os autores constataram que as TIC foram incorporadas ao projeto de ensinar, em que o foco da ação pedagógica está na intencionalidade do processo de ensino e aprendizagem, não na inserção, por si só, das TIC como ferramenta transformadora da prática pedagógica.

Com o olhar voltado para os produtos educacionais, produzidos nos anos de 2010 e 2011 em três cursos de MPECM, que abordavam aspectos da Natureza da Ciência (NdC) no ensino de Física, Vital e Guerra (2014) identificaram 12 produções. Após realizarem uma análise de conteúdo, seguindo a proposta de Bardin, as autoras definiram quatro categorias quanto aos conteúdos dos produtos educacionais: objetivos propostos para a abordagem da NdC no Ensino de Física, literatura que embasa a definição dos objetivos propostos, estratégias didáticas utilizadas pelos autores e dificuldades e obstáculos.

Os 68 produtos educacionais elaborados de 2009 a 2013 no contexto do MPE de Ciência e Tecnologia da Universidade Tecnológica Federal do Paraná - campus Ponto Grossa (UTFPR) foram descritos por Niezer et al. (2015). Ao caracterizarem os produtos em função do formato, da disciplina e do nível de ensino, os autores constataram que embora exista uma variedade de formatos (guias, blogs, vídeos, jogos didáticos), disciplinas (Biologia, Matemática, Química, Informática, Física) e nível de ensino (infantil, fundamental, médio, superior), a maioria dos produtos constitui-se, essencialmente, em Guias Didáticos da disciplina de Matemática direcionados para o Ensino Fundamental.

Tocafundo, Nascimento e Verdejo (2015) categorizaram 48 produtos educacionais na área de Ensino de Física de um MPECM do estado de Minas Gerais. Ao relacionarem os conteúdos dos produtos com o Currículo Básico Comum de Física de Minas Gerais, os autores identificaram o predomínio de conteúdos de Mecânica Clássica, especialmente, Leis de Newton e Aplicações, e pouca ocorrência de temas multidisciplinares, como por exemplo, Energia.

No âmbito do Mestrado Profissional em Matemática em Rede Nacional (PROFMAT), Brena, Font e Lima (2015) identificaram 29 trabalhos de conclusão produzidos em diferentes polos do Estado do Rio Grande do Sul nos anos de 2013 e 2014. Os autores destacam que desse total, 13 trabalhos tratavam da incorporação de conteúdos matemáticos de nível superior na educação básica, sendo os conteúdos de Matemática Discreta e Cálculo os mais recorrentes.

Já Pilatti et al. (2015) mapearam 24 cursos de MPE que possuem como foco principal ou linha de pesquisa o Ensino de Matemática (que não são polos do PROFMAT). Como nove desses, durante o período da pesquisa, não 
disponibilizavam suas produções on-line, os autores analisaram 412 dissertações, defendidas no período de 2004 até meados de 2014, oriundas dos 15 cursos restantes. Como resultado, os autores verificaram que embora haja um crescente número de produtos educacionais, estes concentram-se em propostas de atividades (145), isto é, produtos que apresentam uma sequência de atividades didáticas de conteúdo específico.

Ao analisarem 221 dissertações produzidas nos MPE de Matemática da região Sul do país, defendidas entre 2009 e 2014, Souza et al. (2015) constataram que de todo o corpus da pesquisa, 47 das dissertações não geraram produtos educacionais. Então, os autores categorizaram os 174 produtos educacionais em: sequência didática (guias de atividades de ensino que devem ser realizadas em uma determinada sequência), proposta de formação docente (cursos de formação inicial e continuada), instrumento avaliativo (propostas para avaliação da aprendizagem) e material didático (produção que tem a finalidade de proporcionar a aprendizagem de um determinado conteúdo). Os autores chegaram aos seguintes números: 134 sequências didáticas, 31 materiais didáticos, oito propostas de formação docente e um instrumento avaliativo.

Um estudo tipo "estado da arte" nas dissertações do MPE de Ciências da Universidade de Brasília (UnB) foi feito por Santos, Massi e Villani (2015). Com base nos 99 resumos, referentes às dissertações defendidas no período de 2006 a 2013, os autores observaram uma predominância de produtos educacionais que apresentam recursos didáticos e métodos de ensino nas disciplinas de Física e Química do Ensino Médio. Para os autores, os produtos educacionais desenvolvidos no curso investigado estavam coerentes com a proposta formativa dos MPE, uma vez que focam a pesquisa aplicada em sala de aula.

Com o objetivo de tecer reflexões sobre a importância da pesquisa-ação no contexto de um MPECM, Chisté (2016) analisou cinco dissertações que explicitavam a pesquisa-ação como metodologia adotada dentro de um conjunto de 32 dissertações defendidas até Fevereiro de 2015 no âmbito do curso em questão. Para a autora, nos textos analisados há uma descaracterização da pesquisa-ação, pois muitos aspectos fundamentais foram desconsiderados durante todas as fases da pesquisa (inclusive na estruturação dos projetos). Essa percepção pode explicar, por exemplo, o fato das dissertações analisadas se apresentarem como ações pontuais (casos isolados) que buscaram transformar a realidade escolar dentro do tempo de realização do curso de mestrado.

Especificamente no contexto do Mestrado Profissional em Ensino de Física (MPEF) da Universidade Federal do Rio Grande do Sul (UFRGS) há três estudos sobre os trabalhos de conclusão, sendo todos metodologicamente fundamentados na filosofia da linguagem de Bakhtin. Nascimento, Ostermann e Cavalcanti (2015) analisaram uma dissertação e seu produto educacional, de natureza texto de apoio centrado na Física Clássica. Os autores concluíram que, embora o autor do produto apresente características de um professor reflexivo, isto é, um professor que pesquisa e reflete criativamente as situações-problema para realização de sua prática docente, seu produto educacional apresenta fortes elementos do modelo de especialista técnico, fruto de uma preocupação em atender a demandas do MPEF. Já Souza, Rezende e Ostermann (2016) investigaram a apropriação discursiva do modelo de formação do MPEF em três trabalhos de conclusão. Neste estudo as autoras identificaram nos enunciados analisados um discurso fortemente direcionado ao meio acadêmico. Em outras palavras, o contexto 
formativo do MNPEF, por seguir um modelo de professor especialista técnico, acaba que por influenciar de maneira predominante o discurso dos professores em formação neste curso, pois nos trabalhos de conclusão analisado os professores buscaram atender uma demanda do curso do MPEF, não necessariamente uma demanda oriunda do meio escolar em que exercem a docência. Por fim, Antunes Júnior et al. (2015) investigaram os referenciais teóricos, tentativas de associações e interações entre autores, em 78 dissertações e seus respectivos produtos educacionais defendidas entre os anos de 2004 e 2014. No material analisado, há uma pluralidade de referenciais teóricos utilizados, mas poucas citações das obras originais dos autores dessas teorias. Ainda, nesses trabalhos foi recorrente a associação de teorias que, em essência, não são semelhantes, como é o caso da Teoria da Aprendizagem Significativa de Ausubel com a Teoria Sociocultural de Vygotsky. Para os autores, esse quadro é reflexo da cultura acadêmica do curso, centrada na voz dominante de um livro texto sobre Teorias de Aprendizagem que resume a obra de vários autores e busca estabelecer relações entre as teorias.

\section{(c) entrevistas com egressos de MPECM}

Uma importante vertente para melhor compreender a importância dos MPECM é "ouvir os egressos", em especial, no sentido de buscar elementos que contribuíram para o desenvolvimento profissional (prática docente) dos mesmos. Nesse sentido, há três trabalhos que analisaram os discursos de egressos fundamentados na filosofia da linguagem de Bakhtin, e outros três que adotaram outras abordagens metodológicas.

Suarez e Silva (2015), após identificarem todas as dissertações defendidas nos MPECM do Estado do Rio de Janeiro que tiveram como tema a Educação Ambiental, realizaram uma análise discursiva com dois professores que produziram esses produtos a fim de saber qual o impacto do MPE em suas práticas docentes. Enquanto um dos entrevistados atestou que o MPE contribuiu positivamente para a sua prática docente, o outro afirmou categoricamente que $o$ curso não impactou positivamente sua atividade docente.

Também buscando compreender o impacto nas práticas profissionais, Schäfer, Lima Júnior e Ostermann (2013) analisaram o discurso de dois egressos do MPEF da UFRGS que revelaram uma grande diferença entre a realidade escolar e a formação oferecida no MPEF, de modo que a elaboração do produto educacional acaba por ser realizada como uma demanda do curso, não da escola.

Com o objetivo de investigar a relação entre o desenvolvimento do produto educacional e a utilização do mesmo no ambiente escolar, Moreira e Queiroz (2015) analisaram o discurso de dois egressos de um MPE de Ciências do Rio de Janeiro. Os sujeitos da pesquisa atestaram que enfrentaram dificuldades na implementação do produto educacional no contexto escolar, sendo que acabaram por não utilizá-lo em sua totalidade, mas apenas de maneira superficial.

Seguindo elementos metodológicos da entrevista compreensiva e da multirreferencialidade, Prado, Silva e Araújo (2011) investigaram a atuação de oito professores do Instituto Federal de Educação, Ciências e Tecnologia do Rio Grande do Norte (IFRN) egressos do MPE de Ciências Naturais e Matemática da 
motivações para o ingresso no curso e a atuação profissional após a conclusão. Para os autores, chama a atenção que apenas dois, dos oito entrevistados, declararam utilizar seus produtos com frequência após a conclusão do mestrado.

Ao avaliaram como os estudos e as pesquisas realizados no âmbito do MPECM da Universidade Estadual da Paraíba (UEPB) têm influenciado a prática docente dos egressos desse curso, Moita, Silva e Oliveira (2013), a partir de entrevistas com egressos, diretores e coordenadores das escolas onde os egressos desenvolveram o produto educacional, concluíram que o MPECM da UEPB provocou impactos visíveis na atuação profissional dos egressos nas escolas onde atuam. Os egressos participantes da pesquisa também apontaram como maior contribuição para a prática docente toda a dinâmica envolvida no processo de produção e aplicação do produto educacional. No entanto, os autores verificaram que alguns egressos, após a conclusão do curso, deixaram de utilizar os produtos educacionais em sala.

No último artigo que classificamos nesta categoria, Schuck et al. (2016) investigaram as concepções, culturas, práticas e saberes pessoais e profissionais de oito egressos de um curso de MPE de Ciências Exatas por meio de entrevistas semiestruturadas e posterior análise textual discursiva. Segundo os autores, há uma percepção por parte dos egressos de uma melhoria em suas práticas docentes muito em função do ambiente de formação proporcionado pelo MPE que cursaram. Nesse sentido, os autores destacaram como aspectos fundamentais o ambiente colaborativo e de troca de experiências entre aluno-aluno e alunoprofessor, o trabalho interdisciplinar e o uso das TIC no ensino.

\section{(d) contribuições e potencialidades dos MPECM para a formação continuada de professores}

No âmbito das pesquisas sobre formação continuada de professores, o MPE representa um objeto de estudos e reflexões, assim como um "ambiente fértil" para a coleta de dados de investigações dessa linha de pesquisa. Nessa categoria, identificamos cinco publicações, das quais duas foram desenvolvidas no contexto do MPEF da UFRGS.

O produto educacional, gerado como trabalho de conclusão dos MPECM, como estratégia à educação inclusiva é o cerne das reflexões de Ferreira et al. (2007). Os autores entendem que a elaboração de produtos educacionais voltados para a educação inclusiva de surdos e cegos, por exemplo, pode auxiliar o desempenho do docente na temática inclusão.

Novamente no contexto do MPEF da UFRGS, Ostermann, Prado e Ricci (2008) investigaram as potencialidades da disciplina de Tópicos de Física Moderna e Contemporânea. A partir da análise de questionários respondidos no primeiro e no último dia de aula da disciplina por 14 mestrandos, que também eram professores de Ensino Médio, os autores constataram uma evolução conceitual dos professores em tópicos introdutórios de Física Quântica. Já Moreira e Massoni (2009), durante uma experiência de ensino sobre Física de Partículas, investigaram as concepções epistemológicas dos professores de Ensino Médio que já haviam cursado a disciplina de Epistemologia e Ensino de Física. Para os autores, a sequência de ensino Epistemologia e Física de Partículas propiciou aos mestrandos uma visão do Modelo Padrão através do prisma epistemológico, favorecendo uma reflexão crítica sobre a natureza da ciência. 
O estágio de docência, enquanto processo de formação dos mestrandos do MPE de Ciências na Amazônia da Universidade do Estado do Amazonas (UEA), foi o objeto de reflexão de Pereira e Ghedin (2009). Estes autores afirmam que o estágio de docência não pode ser resumido ao automatismo mecânico e técnico do que aprendemos na Universidade e aplicamos na escola. Pelo contrário, devem ser encarados como momentos de reflexões das inúmeras possibilidades de diálogo que envolvem todos os elementos de uma identidade docente.

Ao descrever os processos coletivos de criação, implementação e avaliação de produtos educacionais, fundamentados na perspectiva da Atividade Orientadora de Ensino, no MPE de Ciências Exatas da Universidade Federal de São Carlos (UFScar), Sousa (2013) destacou que nessa proposta o produto educacional foi resultado de um processo reflexivo e contextualizado acerca dos saberes teóricos e experienciais, refletindo, assim, a essência da proposta formativa dos MPE.

\section{(e) discussão sobre os Mestrados Profissionais em Rede Nacional}

Uma medida política da CAPES que acelerou a expansão dos cursos de MPE foi os programas de Mestrado Profissional em Rede Nacional ${ }^{4}$. Sobre estes programas encontramos os estudos de Cladatto, Pavanello e Fiorentini (2016) sobre o Programa de Mestrado Profissional em Matemática em Rede Nacional (PROFMAT) e de Rebeque e Ostermann (2015) sobre o Mestrado Nacional Profissional em Ensino de Física (MNPEF).

No primeiro caso, os autores investigaram alguns dos documentos legais do PROFMAT, tais como: projeto acadêmico, livros-texto, currículo nacional, além de dados oriundos de observação de aulas, como por exemplo questionários respondidos por acadêmicos e avaliações presenciais. Com base em uma análise na perspectiva processual e descentralizadora, os autores concluíram que o PROFMAT objetiva uma formação técnica e acadêmica desconectada de uma formação didático-pedagógica, sobretudo pelo fato do currículo nacional não estar diretamente vinculado à prática do professor de Matemática da educação básica.

Quanto ao estudo sobre o MNPEF, fundamentados em um conjunto de ideias e propostas globais que visam melhorar a formação continuada de professores, Rebeque e Ostermann (2015) analisaram as normas e o currículo do programa em rede da Física. Para os autores, as concepções e práticas de formação intrínsecas ao MNPEF alinham-se ao chamado modelo de "treinamento" (cursos padronizados ministrados por especialistas que se concentram na resolução de problemas genéricos), mostrando-se descontextualizadas dos estudos até então realizados sobre formação continuada de professores ou sobre os cursos de MPE.

\section{(f) investigações de vários aspectos no contexto específico de cursos de MPECM}

Este último grupo, formado por sete publicações, reúne pesquisas sobre cursos específicos de MPECM, ou seja, tomam como objeto de investigação vários aspectos que envolvem um determinado curso de MPECM.

Sobre o MPECM da Universidade Cruzeiro do Sul (UNICSUL), Araújo e Amaral (2007) descreveram a proposta, a estrutura curricular e, com base na análise de questionários respondidos por estudantes do curso, comentaram os problemas, as 
dificuldades e os impactos provocados por este curso na atividade docente dos sujeitos da pesquisa.

Um estudo descritivo do MP em Educação Matemática da Pontifícia Universidade Católica de São Paulo (PUC/SP) foi apresentado por Pires e Igliori (2013). Ao reunirem documentos e entrevistas com os mestrandos, as autoras descreveram o processo de criação e evolução, o perfil dos estudantes e os temas abordados nos 100 primeiros trabalhos de conclusão. Na avaliação das autoras, o curso estudado está consistente com a proposta de um curso de MPE, isto é, focado na formação em conteúdos matemáticos, didáticos e curriculares pertinentes à realidade escolar vivenciada por seus estudantes.

Grassi et al. (2016) entrevistaram nove docentes inseridos no contexto de um MPE de Ciências Exatas com o objetivo de investigarem as competências, as habilidades e as perspectivas desses sujeitos no que se refere às tomadas de decisões sobre a organização curricular, planejamentos metodológicos de ensino e aprendizagem, processos de avaliação e ações de (re)construção. De acordo com a análise textual discursiva empreendida pelos autores, foi possível identificar ações desses docentes na direção do aperfeiçoamento do curso em questão, em especial, na estrutura curricular a partir da inserção e compartilhamento de disciplinas que buscam aproximar o saber das pesquisas com a atividade docente.

Quatro publicações tomaram como objeto de estudo o MPEF da UFRGS:

As pesquisas de Schäfer e Ostermann (2011, 2013a e 2013b) consistiram em entrevistas com alunos-professores (AP) em diferentes situações no curso (ingressante e regulares) e egressos, posteriormente analisadas segundo pressupostos bakhtinianos. De modo geral, ao investigarem o contexto de formação do MPEF e os impactos provocados no trabalho docente dos AP, as autoras concluíram que este curso favorece os saberes da formação profissional (acadêmicos) sem a devida articulação com os saberes da formação inicial e os saberes oriundos da atuação docente nas escolas (SCHÄFER e OSTERMANN, 2013a). Outra constatação das autoras é que a maioria dos AP se encontra sob forte influência da racionalidade técnica (isto é, sua atuação docente se concentra na aplicação de um conhecimento teórico e técnico), sendo que o MPEF, enquanto curso de formação continuada, acaba que por reforçar esse tipo de racionalidade (SCHÄFER e OSTERMANN, 2013b).

Por fim, a pesquisa de Barolli, Villanni e Maia (2015) buscou refletir sobre o processo de constituição do curso investigado, mediante sua reconstrução histórica. A partir de entrevistas com nove docentes do curso, seguida de uma análise narrativa elaborada com base no desenvolvimento grupal de Kaës, os autores destacam o período inicial de sucesso do curso e, posteriormente, um período de crise, fruto de divergências de diferentes naturezas, que, aliado à criação do programa em Rede Nacional (MNPEF), contribuiu para o encerramento deste curso no ano de 2015.

\section{CONSIDERAÇÕES FINAIS}

O grande volume de publicações em periódicos e o crescente número de participantes e trabalhos publicados nas atas do ENPEC (Delizoicov, Slongo e Lorenzetti, 2013) demonstram a consolidação da área de pesquisa em ECM no Brasil. Porém, pensando no crescimento desta área no SNPG, sobretudo através 
da criação de cursos de MPECM, percebemos que a produção acadêmica sobre esta questão ainda é pequena, com um total de 37 publicações na última década em 20 revistas nacionais e internacionais e nas atas ENPEC que consultamos (ver tabelas 2 e 3). Por outro lado, o MPECM passou a ter maior destaque nas pesquisas nos últimos três anos, período em que 25 pesquisas foram publicadas, o que nos indica que as atenções da academia aos poucos estão se voltando para este tema.

Esta produção está fortemente concentrada em poucos periódicos, pois dos 20 que consultamos apenas nove publicaram pesquisas sobre o MPECM. No caso da revisão bibliográfica empreendida por Villani et al. (2017), também sobre os MPECM, os autores identificaram 26 pesquisas sobre o tema que foram publicadas em 13 diferentes periódicos durante os anos de 2000 e 2015. No entanto, metade destas pesquisas foram publicadas em periódicos de menor circulação acadêmica (qualis B2, B3 e B4, segundo classificação da CAPES).

Nos textos que revisamos, percebemos a forte tendência em estudos sobre as dissertações e os produtos educacionais oriundos dos MPECM. Estes estudos se concentraram na identificação e categorização, deixando de realizar uma investigação mais ampla sobre a qualidade, a utilização (pelos próprios autores e/ou por outros professores) e a disseminação desses materiais. Dado o grande volume de produtos educacionais que estão sendo produzidos nos MPECM, assim como nos programas em rede da Matemática e da Física, este assunto tem potencial para ser bastante explorado em pesquisas futuras.

Outro fato que nos chamou a atenção foi a concentração em pesquisas sobre os cursos de MPE de Física e de Matemática e a ausência sobre os de Biologia de Química. As disciplinas de Física e Matemática, aliás, possuem seus próprios programas em Rede Nacional, mas que foram poucos explorados pelas publicações aqui citadas 5 . Há de se destacar que dez pesquisas foram desenvolvidas no âmbito do MPEF da UFRGS, pioneiro no Brasil na modalidade Ensino de Física, cujo currículo serviu de modelo para o MNPEF. Essencialmente, essa produção é fruto de um grupo de pesquisa, vinculado ao Programa de Pós-Graduação em Ensino de Física (PPGEnFis) da UFRGS, que há algum tempo está realizando estudos sobre o MPEF da UFRGS. Além de artigos e trabalhos completos, este grupo já produziu uma tese de doutorado (SCHÄFER, 2013) e duas dissertações de mestrado acadêmico (SOUZA, 2015 e NASCIMENTO, 2016).

Por fim, podemos dizer que essa produção acadêmica tem sido importante não somente para o levantamento de tendências, mas para a identificação de lacunas, como por exemplo, estudos sobre as diretrizes e os currículos dos MPECM, sobre os recentes programas em rede nacional, e, principalmente, acerca do MPECM enquanto instrumento de política pública de formação continuada de professores. Nesse sentido, todo esse contexto de expansão dos cursos de MPECM, as dissertações e os produtos educacionais, o perfil dos egressos, entre tantas outras possibilidades, apresentam-se para os pesquisadores da área ECM como uma problemática rica e fértil a ser investigada.

\section{AGRADECIMENTOS}

À Coordenação de Aperfeiçoamento de Pessoal de Nível Superior (CAPES) que, por meio do Edital Observatório da Educação (OBEDUC), apoiou financeiramente a presente pesquisa. 


\title{
The professional master's degrees in Science and Math teaching in Brazil: a subject little explored in the literature.
}

\begin{abstract}
We present in this paper a review of literature regarding the professional master's degrees in Science and Math teaching in Brazil. Therefore, we examined the publications of the last decade in 20 national and international journals and at the National Meeting of Research in Science Education. Inside the large volume of texts consulted, we found 37 publications, indicating that this subject is still little explored in the literature. This review was important to identify trends, such as studies that characterize the dissertations developed in these courses and research on specific courses of Physics and Math, but not about Biology and Chemistry courses. On the other hand, there is a lack of research of the guidelines and curriculum of courses in Science and Math teaching, on recent programs on National Network and the role of these courses as public policy in service teacher education.
\end{abstract}

KEYWORDS: professional master's degrees, Science and Math teaching, in service teacher education. 


\section{NOTAS}

1 Dez anos após a publicação da Portaria n. 47/1995, a Revista Brasileira de PósGraduação (RBPG) lançou uma edição temática (v. 2, n. 4, 2005) com 12 artigos que apresentaram, em seu conjunto, um breve histórico do caminho percorrido e a situação do MP no Brasil na época da publicação. Para consultar a referida edição acesse: http://ojs.rbpg.capes.gov.br/index.php/rbpg/issue/view/4.

2 Os dados quantitativos dos programas de pós-graduação podem ser acessados em tempo real na Plataforma Sucupira, base de dados do SNPG, cujo endereço eletrônico é https://sucupira.capes.gov.br/sucupira/public/index.jsf.

Todos os dados quantitativos dos cursos de mestrado aqui apresentados foram obtidos no dia 30 de Março de 2017. Como as informações na Plataforma Sucupira são atualizadas em tempo real, esses números não serão os mesmos na data de publicação deste artigo.

3 Nos periódicos que consultamos nossa busca foi feita ou na base de dados SciELO (http://www.scielo.br/) ou no acervo dos próprios periódicos que não pertencem a essa base. Quanto às Atas do ENPEC, todas são disponibilizadas pela Associação Brasileira de Pesquisa em Educação em Ciências (ABRAPEC), entidade que promove o evento (http://abrapecnet.org.br/wordpress/pb/atas-dos-enpecs/).

4 Para termos uma ideia da rápida expansão de cursos de MPE através dos programas em rede nacional, até o final do ano de 2016 as áreas de Matemática, Letras, Física, Artes, Administração Pública, História e Gestão e Recursos Hídricos criaram seus próprios programas. Maiores informações sobre cada programa estão disponíveis em http://www.capes.gov.br/educacao-a-distancia.

5 Em 23 de Novembro de 2015 foi divulgada uma chamada pública para a adesão ao Programa de Mestrado Profissional em Ensino de Biologia em Rede Nacional (ProfBio). No entanto, até o dia 30 de Março de 2017 este programa não figurava entre os mestrados em rede nacional no site da CAPES (link da nota anterior).

\section{REFERÊNCIAS}

ANTUNES JÚNIOR, E.; REBEQUE, P. V.; OSTERMANN, F.; CAVALCANTI, C. J. H. Sobre dissertações no contexto do Mestrado Profissional em Ensino de Física. In: $X$ ENPEC - Encontro Nacional de Pesquisa em Educação em Ciências, Águas de Lindóia. Anais... Águas de Lindóia: ABRAPEC, FP-34, 2015.

ARAÚJO, M. S. T.; AMARAL, L. H. O Mestrado Profissional em Ensino de Ciências e Matemática da UNICSUL e a formação docente: contribuições e perspectivas de desenvolvimento profissional dos estudantes. In: VI ENPEC - Encontro Nacional de Pesquisa em Educação em Ciências, Florianópolis. Anais... Florianópolis: ABRAPEC, 2007. 
BRENDA, A.; FONT, V.; LIMA, V. M. R. Propuestas de incorporación de contenidos matemáticos de nivel superior en la educación básica: un estúdio de los trabajos de curso del Máster Profesional en Matemáticas en la Red Nacional. Revista Brasileira de Ensino de Ciência e Tecnologia, Curitiba, v. 8, n. 3, p. 136-148, 2015.

CALDATTO, M. E.; PAVANELLO, R. M.; FIORENTINI, D. O PROFMAT e a formação do professor de Matemática: uma análise curricular a partir de uma perspectiva processual e descentralizadora. Boletim de Educação Matemática, Rio Claro, v. 30, n. 56, p. 906-925, 2016.

CAPES. Coordenação de Aperfeiçoamento de Pessoal de Nível Superior. Mestrado no Brasil - a situação e uma nova perspectiva. Infocapes, Brasília, v. 3, n. 3-4, p. 18-21, 1995.

CAPES. Coordenação de Aperfeiçoamento de Pessoal de Nível Superior. Critérios de Avaliação Trienal 2004/2006 - Área de Ensino de Ciências e Matemática. Brasília, 2007. Disponível em <https://www.capes.gov.br/images/stories/downlo ad/avaliacao/CA2007 CienciasMatematica.pdf>. Acesso em: 30 mar. 2017.

CAPES. Coordenação de Aperfeiçoamento de Pessoal de Nível Superior. Relatório de Avaliação Trienal 2007/2009 - Área de Ensino de Ciências e Matemática. Brasília, 2010b. Disponível em <http://trienal.capes.gov.br/wp-content/uploads/ 2011/01/ENSINO-DE-CM-RELAT\%C3\%93RIO-DE-AVALIA\%C3\%87\%C3\%830-FINALjan11.pdf>. Acesso em: 30 mar. 2017.

CAPES. Coordenação de Aperfeiçoamento de Pessoal de Nível Superior. Relatório de Avaliação Trienal 2010/2012 - Área de Ensino. Brasília, 2013. Disponível em <http://avaliacaotrienal2013.capes.gov.br/documento-de-area-e-comissao>. Acesso em: 30 de mar. 2017.

CARNEIRO, V. C. G. Contribuições para a formação do professor de Matemática pesquisador nos Mestrados Profissionalizantes na Área de Ensino. Boletim de Educação Matemática, Rio Claro, v. 21, n. 29, p. 199-222, 2008.

CARVALHO, R. B.; OLIVEIRA, R. L.; SILVA, M. G. L.; BARROS, W. I. T. Caracterização dos Produtos das Dissertações em Ensino de Ciências Naturais e Matemática da UFRN, Brasil. In: VIII ENPEC - Encontro Nacional de Pesquisa em Educação em Ciências, Campinas. Anais... Campinas: ABRAPEC, 647-2, 2011. 
CHISTÉ, P. S. Pesquisa-ação em mestrado profissionais: análise de pesquisas de um programa de pós-graduação em ensino de ciências e de matemática. Ciência \& Educação, Bauru, v. 22, n. 3, p. 789-808, 2016.

DELIZOICOV, D.; SLONGO, I. I. P.; LORENZETTI, L. Um panorama da pesquisa em educação em ciências desenvolvida no Brasil de 1997 a 2005. Revista Electrónica de Enseñanza de las Ciencias, Vigo, v. 12, n. 3, p. 459-480, 2013.

FERREIRA, A. C.; COUTINHO, F. A.; SCHEID, E. G.; CHAVES, A. C. L.; DICKMAN, A. G. O Mestrado Profissional em Ensino de Ciências e Matemática e a questão do produto como estratégia à educação inclusiva. In: VI ENPEC - Encontro Nacional de Pesquisa em Educação em Ciências, Florianópolis. Anais... Florianópolis: ABRAPEC, 2007.

GRASSI, M. H.; MARCHI, M. I.; SCHUCK, R. J.; MARTINS, S. N. Docência em mestrado profissional: registros de percepções e práticas em (re)construção. Revista Brasileira de Educação, Rio de Janeiro, v. 21, n. 66, p. 681-698, 2016.

KRUMMENAUER, W. L.; COSTA, S. S. C.; SILVEIRA, F. L. Uma experiência de Ensino de Física contextualizada para a Educação de Jovens e Adultos. Ensaio: pesquisa em educação em ciências, Belo Horizonte, v. 12, n. 2, p. 69-82, 2010.

MOITA, F. G. S. C.; SILVA, A.; OLIVEIRA, K. O mestrado profissional como formação continuada e as metas educacionais vigentes. In: IX ENPEC - Encontro Nacional de Pesquisa em Educação em Ciências, Águas de Lindóia. Anais... Águas de Lindóia: ABRAPEC, 1144-1, 2013.

MOREIRA, M. A. A área de Ensino de Ciências e Matemática na CAPES: panorama 2001/2002 e critérios de qualidade. Revista Brasileira de Pesquisa em Educação em Ciências, São Paulo, v. 2, n. 1, p. 36-59, 2002.

MOREIRA, M A. O mestrado (profissional) em ensino. Revista Brasileira de PósGraduação, Brasília, n. 1, p. 131-142, 2004.

MOREIRA, M. A.; MASSONI, N. T. Física de Partículas y visiones epistemológicas contemporáneas em la formación postgraduada de professores de Física.

Experiências em Ensino de Ciências, Cuiabá, v. 4, n. 1, p. 57-64, 2009.

MOREIRA, M. A.; NARDI, R. O mestrado profissional na área de Ensino de Ciências e Matemática: alguns esclarecimentos. Revista Brasileira de Ensino de Ciência e Tecnologia, Curitiba, v. 2, n. 3, p. 1-9, 2009. 
NASCIMENTO, M. M. Análise de produtos educacionais desenvolvidos no âmbito de um Mestrado Profissional em Ensino de Física. Dissertação (Mestrado em Ensino de Física) - Programa de Pós-Graduação em Ensino de Física. Universidade Federal do Rio Grande do Sul, Porto Alegre, 2016.

NASCIMENTO, M. M.; OSTERMANN, F.; CAVALCANTI, C. J. H. Análise de um produto educacional desenvolvido no âmbito de um Mestrado Profissional em Ensino de Física. In: X ENPEC - Encontro Nacional de Pesquisa em Educação em Ciências, Águas de Lindóia. Anais... Águas de Lindóia: ABRAPEC, FP-13, 2015.

NIEZER, T. M.; FABRI, F.; FRASSON, A. C.; PILATTI, L. A. Caracterização dos produtos desenvolvidos por um programa de Mestrado Profissional da área de Ensino de Ciências e Tecnologia. Revista Brasileira de Ensino de Ciência e Tecnologia, Curitiba, v. 8, n. 3, p. 1-30, 2015.

OLIVEIRA, V.; VEIT, E. A.; ARAUJO, I. S. Relato de experiência com os métodos Ensino sob Medida (Just-in-time Teaching) e Instrução pelos Colegas (Peer Instruction) para o ensino de tópicos de Eletromagnetismo. Caderno Brasileiro de Ensino de Física, Florianópolis, v. 32, n.1, p. 180-206, 2015.

OSTERMANN, F.; PRADO, S. D.; RICCI, T. Investigando a aprendizagem de professores de Física acerca do fenômeno de interferência quântica. Ciência \& Educação, Bauru, v. 14, n. 1, p. 35-54, 2008.

OSTERMANN, F.; REZENDE, F. Projetos de desenvolvimento e de pesquisa na área de ensino de ciências e matemática: uma reflexão sobre os mestrados profissionais. Caderno Brasileiro de Ensino de Física, Florianópolis, v. 26, n. 1, p. 66-80, 2009.

PEREIRA, W. S. C.; GHEDIN, E. Estágio docência na formação de professores mestres para o Ensino de Ciências na Amazônia. In: VII ENPEC - Encontro Nacional de Pesquisa em Educação em Ciências, Florianópolis. Anais... Florianópolis: ABRAPEC, 2009.

PILATTI, L. A.; COSTA, J. M.; SCHIRLO, A. C.; DA SILVA, S. C. R.; PINHEIRO, N. A. M.; FRASSON, A. C. Mestrado Profissional em Ensino de Matemática: identificação de seus produtos educacionais. Revista Brasileira de Pós-Graduação, Brasília, v. 12, n. 28, p. 335-356, 2015.

PIRES, C. M. C.; IGLIORI, S. B. C. Mestrado Profissional e desenvolvimento profissional do professor de Matemática. Ciência \& Educação, Bauru, v. 19, n. 4, p. 1045-1068, 2013. 
PRADO, M. R. M.; SILVA, M. G. L.; ARAÚJO, M. F. F. A formação pós-graduada em Ensino de Ciências Naturais e Matemática de docentes do IFRN: implicações na atuação docente. In: VIII ENPEC - Encontro Nacional de Pesquisa em Educação em Ciências, Campinas. Anais... Campinas: ABRAPEC, 350-1, 2011.

REBEQUE, P. V.; OSTERMANN, F. Reflexões sobre o Mestrado Nacional Profissional em Ensino de Física. In: X ENPEC - Encontro Nacional de Pesquisa em Educação em Ciências, Águas de Lindóia. Anais... Águas de Lindóia: ABRAPEC, FP27, 2015.

REZENDE, F.; OSTERMANN, F. O protagonismo controverso dos mestrados profissionais em ensino de ciências. Ciência \& Educação, Bauru, v. 21, n. 3, p. 543-558, 2015.

SANTOS, S. R. M.; MARTINS, H. G.; PUGGIAN, C.; DA COSTA, P. M. D. Didáticas específicas, novas tecnologias e formação de professores para o ensino das Ciências na baixada fluminense: a experiência do mestrado profissional da Universidade do Grande Rio. Revista Brasileira de Pós-Graduação, Brasília, v. 9, n. 16, p. 115-138, 2012.

SANTOS, V. F. D.; MASSI, L.; VILLANI, A. O estado da arte das dissertações do Mestrado Profissional em Ensino de Ciências da Universidade de Brasília. In: X ENPEC - Encontro Nacional de Pesquisa em Educação em Ciências, Águas de Lindóia. Anais... Águas de Lindóia: ABRAPEC, FP-27, 2015.

SCHÄFER, E. D. A. Impacto do Mestrado Profissional em Ensino de Física da UFRGS na prática docente: um estudo de caso. 2013. Tese (Doutorado em Ensino de Física) - Programa de Pós-Graduação em Ensino de Física. Universidade Federal do Rio Grande do Sul, Porto Alegre, 2013.

SCHÄFER, E. D. A.; LIMA JÚNIOR, P.; OSTERMANN, F. Documentando opiniões e críticas dos alunos de um mestrado profissional em Ensino de Física. In: IX ENPEC - Encontro Nacional de Pesquisa em Educação em Ciências, Águas de Lindóia. Anais... Águas de Lindóia: ABRAPEC, 841-1, 2013.

SCHÄFER, E. D. A.; OSTERMANN, F. O impacto de um Mestrado Professional em Ensino de Física na prática docente de seus alunos: uma análise bakhtiniana sobre os saberes profissionais. Ensaio: pesquisa em educação em ciências, Belo Horizonte, v. 15, n. 2, p. 87-103, 2013a.

SCHÄFER, E. D. A.; OSTERMANN, F. Autonomia professional na formação de professores: uma análise de entrevistas realizadas num Mestrado Profissional em 
SCHUCK, R. J.; MARTINS, S. N.; MARCHI, M. I.; GRASSI, M. H. Ensino em mestrado profissional de Ciências Exatas: concepções e saberes de professores. Revista Brasileira de Pós-Graduação, Brasília, v. 13, n. 31, p. 491-506, 2016.

SILVA NETO, J.; OSTERMANN, F.; PRADO, S. D. O tema da dualidade ondapartícula na educação profissional em radiologia médica a partir da simulação do interferômetro de Mach-Zehnder. Revista Brasileira em Ensino de Física, São Paulo, v. 33, n.1, p. 1401, 2011.

SOUZA, M. C. Quando professores que ensinam Matemática elaboram produtos educacionais, coletivamente, no âmbito do Mestrado Profissional. Boletim de Educação Matemática, Rio Claro, v. 27, n. 47, p. 875-899, 2013.

SOUZA, J. Apropriação discursiva de modelos de formação docente em trabalhos de conclusão de um Mestrado Profissional em Ensino de Física. Dissertação (Mestrado em Ensino de Física) - Programa de Pós-Graduação em Ensino de Física. Universidade Federal do Rio Grande do Sul, Porto Alegre, 2015.

SOUZA, J.; REZENDE, F.; OSTERMANN, F. Apropriação discursiva de modelos de formação docente em trabalhos de conclusão de um Mestrado Profissional em Ensino de Física. Ensaio: pesquisa em educação em ciências, Belo Horizonte, v. 18, n. 2, p. 171-199, 2016.

SOUZA, M. J. F. S.; MELO, T. F. O.; VILELA, L. G. A. F.; RIBEIRO, E. A. L.; DIOGO, R. C.; GUIMARÃES, C. S. Análise dos produtos de programas de mestrado profissional: um recorte envolvendo o Ensino de Matemática na região Sul do Brasil. In: X ENPEC - Encontro Nacional de Pesquisa em Educação em Ciências, Águas de Lindóia. Anais... Águas de Lindóia: ABRAPEC, POL-03, 2015.

SUAREZ, A. P.; SILVA, A. M. T. B. A educação ambiental nos mestrados profissionais do Rio de Janeiro: que voz ecoa na sala de aula? In: X ENPEC Encontro Nacional de Pesquisa em Educação em Ciências, Águas de Lindóia. Anais... Águas de Lindóia: ABRAPEC, EAM-02, 2015.

TOCAFUNDO, R. D.; NASCIMENTO, S. S.; VERDEJO, A. M. Análise de produtos de um Mestrado Profissional em Ensino de Ciências e Matemática: panorama das produções para o Ensino de Física. In: X ENPEC - Encontro Nacional de Pesquisa em Educação em Ciências, Águas de Lindóia. Anais... Águas de Lindóia: ABRAPEC, FP-27, 2015.

VILLANI, A. Mestrado Profissional em Ensino de Ciências e Matemática; uma interpretação. Revista Ibero-Americana de Estudos em Educação, Araraquara, v. 11, n. especial 1, p. 418-433, 2016. 
VILLANI, A.; BAROLLI, E.; MAIA, J. O.; MASSI, L.; SANTOS, V. F. D.;NASCIMENTO, W. E. Mestrados Profissionais em Ensino de Ciências: estrutura, especialidade, efetividade e desenvolvimento profissional docente. Investigações em Ensino de Ciências, Porto Alegre, v. 22, n. 1, p.127-161, 2017.

VITAL, A.; GUERRA, A. A natureza da ciência no ensino de Física: estratégias didáticas elaboradas por professores egressos do mestrado profissional. Caderno Brasileiro de Ensino de Física, Florianópolis, v. 31, n. 2, p. 225-257, 2014.

WOLFF, J. F. S.; MORS, P. M. Relatividade no Ensino Médio: uma experiência com motivação na História. Experiências em Ensino de Ciências, Cuiabá, v. 1, n.1, p. 14-22, 2006.

Recebido: 2016-07-01

Aprovado: 2017-05-22

DOI: $10.3895 /$ rbect.v10n2.4386

Como citar:

REBEQUE, P. V.; OSTERMANN, F.; VISEU, S. Sobre os Mestrados Profissionais em Ensino de Ciências e Matemática no Brasil: um tema pouco explorado na literatura. Revista Brasileira de Ensino de Ciência e Tecnologia, v. 10, n. 2, 2017. Disponível em: <https://revistas.utfpr.edu.br/rbect/article/view/4386>. Acesso em: $x x x$.

Correspondência: Av. Osvaldo Aranha, 540, 95700-206 - Bento Gonçalves - Rio Grande do Sul Direito autoral: Este artigo está licenciado sob os termos da Licença Creative Commons-Atribuição 4.0 Internacional. 